\title{
"A survey study of the impact of COVID-19 on women at high risk for infant mortality in Central Indiana"
}

Tanvi Asthana ${ }^{1}$, Qing Tang ${ }^{1,2}$, Matt Hodges ${ }^{1}$, Debra Litzelman ${ }^{1,3,4}$

${ }^{1}$ Indiana University School of Medicine, ${ }^{2}$ Department of Biostatistics, ${ }^{3}$ Indiana University Health, ${ }^{4}$ Regenstrief Institute

\section{Background/Objective:}

The WeCare program aims to reduce infant mortality through the community health worker model by assisting high risk women of child-bearing age. The COVID-19 pandemic introduced additional challenges for women already at high risk factor for infant mortality (IM). The aim of this survey study is to assess the impact of the COVID-19 pandemic in this population.

\section{Methods:}

Enrolled WeCare participants were given an optional COVID-19 phone survey (modified from the leDEA survey*) between August 2020 and May 2021 to assess the impact of COVID-19 on their daily lives, income, food security, and mental health. Trained research assistants and community health workers administered surveys. Verbal consent was obtained over the phone. Data was entered into REDCap. From REDCap, data was exported to SAS version 9.4 to calculate descriptive statistics and chi square tests.

\section{Results:}

Fifty-six primarily women of color (68\%) completed the survey. Sixty-two percent of women lived in high-risk zip codes for infant mortality in Central Indiana. Fifty-seven percent of women worked prior to the outbreak. Forty-eight percent of the working women lost income, and seventy percent of women worked jobs requiring interaction with the public. These women experienced greater food insecurity (38\%), depression (24\%), and anxiety $(31 \%)$ compared to before the pandemic $(p<0.001)$.

\section{Conclusion/Discussion:}

The demographics and residence of those interviewed were representative of the WeCare cohort (NS differences in race/ethnicity/age). In comparing the prevalence of food insecurity, depression and anxiety between the survey sampled during covid and prior to covid, all factors were significantly increased. These vulnerable women (many pregnant or postpartum), often major breadwinners in their household, held jobs putting them at high risk for covid infections, and lost these high-risk jobs at an alarming rate. These data confirm the detrimental impact of COVID-19, especially on a population already at high risk for IM.

Acknowledgement of original authors of the *leDEA survey: Suzanne Goodrich, Michael Scanlon, Leslie Enane, Kara Wools-Kaloustian 
